\title{
Separação-individuação aos pais e bem-estar psicológico em jovens adultos: Papel mediador da personalidade
}

\author{
Maria Helena Morais ${ }^{1}$ \& Catarina Pinheiro Mota ${ }^{1,2}$ \\ ${ }^{1}$ Departamento de Educação e Psicologia, Universidade de Trás-os-Montes e Alto Douro \\ ${ }^{2}$ Centro de Psicologia da Universidade do Porto
}

\begin{abstract}
Resumo: As ligações estabelecidas precocemente com as figuras parentais assumem um papel importante no desenvolvimento da individuação dos jovens adultos, na construção da sua personalidade e na manifestação do bem-estar psicológico. 0 presente estudo tem como objetivo estudar o papel preditor da separação-individuação aos pais e da personalidade no bem-estar psicológico e, ainda, testar o papel mediador da personalidade na associação anterior. A amostra é composta por 948 sujeitos com idades entre os 18 e os 30 anos de idade. Os resultados indicam que a separação-individuação aos pais positiva prediz positivamente o bem-estar psicológico e a personalidade emocionalmente ajustada. Por outro lado, o neuroticismo prediz negativamente o bem-estar psicológico. A personalidade emocionalmente ajustada assume um papel de mediação total na associação entre a separação-individuação na mãe positiva e no bem-estar psicológico. Por sua vez, a personalidade emocionalmente ajustada assumiu uma mediação parcial na associação entre a separação-individuação no pai positiva e o bem-estar psicológico.
\end{abstract}

Palavras-chave: Separação-individuação; personalidade; bem-estar; jovens adultos.

Separation-individuation to parents processes and psychological well-being in young adults: the mediational role of personality: The quality of the early relationships with parents have an important role in the development of the individuation of young adults in the construction of their personality and in the psychological well-being. This study aim to study the predictor role of separation-individuation to parents and the personality in the psychological well-being. It also tests the mediator role of personality in the previous association. The sample is composed by 948 people with ages between 18 and 30. The results show that the separation-individuation to parents predicts positively the psychological well-being and the adjusted emotional personality. The neuroticism predicts negatively the psychological well-being. The adjusted emotional personality assumes a total mediator role in association between the positive separation- individuation mother and the psychological well-being. The adjusted personality assumes a partial mediation in association between the positive separation-individuation father and the psychological well-being.

Keywords: Separation-individuation; personality; well-being; young adults.

A teoria da Vinculação preconiza que a qualidade das experiências precoces estabelecidas com as figuras parentais tem um papel relevante no desenvolvimento afetivo da criança (Bowlby, 1973). 0 vínculo é definido como um laço que o indivíduo estabelece com as figuras significativas ao longo da sua vida. Este é fortalecido através da proximidade com as figuras significativas, promotora de segurança e apoio (Bowlby, 1969). As figuras que proporcionam segurança e apoio são consideradas bases seguras para o indivíduo, sendo através do reconhecimento das figuras de vinculação como bases seguras que o indivíduo explora o mundo exterior com segurança (Ainsworth 1967, 1989). A experiência dos vínculos com as figuras primordiais possibilita a elaboração de modelos internos dinâmicos que se traduzem na perceção que o indivíduo tem acerca de si próprio e dos outros (Bowlby 1973, 1980). A vinculação às figuras parentais assume ainda um papel significante no desenvolvimento da construção da identidade dos jovens (Wilkinson, 2004). A individuação possibilita a consolidação da singularidade do indivíduo, envolvendo o desejo de o jovem ser autónomo e, por outro lado, a apreensão em enfrentar o desconhecido (Fleming, 2005; Mahler, Pine \& Bergman, 1975). A individuação passa pela separação emocional das figuras parentais (Fleming, 2005), pelo que ambos os processos ocorrem em paralelo (Meeus, Iedema, Maassen, \& Engel, 2005). A perceção das figuras parentais como afetuosas e disponíveis auxilia o desenvolvimento do

${ }^{1}$ Morada de correspondência: Catarina Pinheiro Mota. Departamento de Educação e Psicologia, ECHS - Polo I, Universidade de Trás-os-Montes e Alto Douro, 5000-800 Vila Real, Portugal. E-mail: catppmota@utad.pt. Esta investigação foi financiada pelo Centro de Psicologia da Universidade do Porto, Fundação para a Ciência e Tecnologia (FCT UIDB/00050/2020). 
processo de individuação (Boles, 1999). Por outro lado, as figuras parentais intrusivas, que desencorajam os jovens a ter as suas próprias ideias, dificultam o desenvolvimento da individuação (Barber, 2002). As alterações sociais, económicas e demográficas a que os "adultos emergentes" são expostos (Arnett, 2006), o crescente investimento na vida profissional e a saída tardia da casa dos pais parecem dificultar também a consolidação da individuação (Arnett 2006, 2007).

0 desenvolvimento de uma personalidade ajustada encontra-se de forma significativa relacionada com os vínculos estabelecidos com as figuras significativas (Bowlby, 1969) e, por sua vez, com o processo de separação-individuação (Kins, Soenens, \& Beyers, 2011), em que se verificam mudanças na estrutura psíquica (Blos, 1996). A personalidade é definida pelos padrões intemporais (atitudes e comportamentos) que caraterizam a forma individual que os indivíduos tendem a agir em diferentes situações (Roberts, 2009). A personalidade divide-se em duas esferas, uma que se associa com a adaptação e o ajustamento da pessoa, e outra associada com vulnerabilidade emocional (Eisenberg, Fabes, Guthrie, \& Reiser, 2000). 0 modelo fatorial da personalidade sustentado em cinco fatores indica a compreensão e análise dos traços de personalidade que estão agrupados por dimensões, nomeadamente a extroversão, amabilidade, conscienciosidade, neuroticismo e abertura à experiência (Nunes, Hutz, \& Giacomoni, 2009). A extroversão remete para a assertividade, sociabilidade, expressividade, estando associada a emoções positivas. A amabilidade carateriza-se pela filantropia, honestidade, altruísmo, flexibilidade e bondade (Nunes \& Hutz, 2007). A conscienciosidade associa-se à sensatez, responsabilidade, competência e perseverança (Nunes \& Hutz, 2007; Shanahan, Hill, Roberts, Eccles, \&Friedman, 2014). A abertura à experiência prende-se com a curiosidade por coisas novas e aventura. 0 neuroticismo diz respeito aos afetos negativos, como a ansiedade, vulnerabilidade, tensão, tristeza, depressão e raiva (Nunes \& Hutz, 2007; Vasconcellos \& Hutz, 2008).

A personalidade associa-se de forma significativa como o bem-estar psicológico (Novo, 2005; Ryff, 1989; Ryff \& Keyes, 1995). O bem-estar psicológico observa-se através da maneira em que o sujeito se relaciona consigo, com os outros e com as ocorrências do dia a dia (Gómez-Baya, Lucia-Casademunt, \& Salinas-Pérez, 2018; Pinto, 2009). Um estudo de Kokko, Tolvanen e Pulkkinen (2013), em que a amostra foi composta por adultos com idades compreendidas entre os 33 e os 50 anos de idade, objetivou examinar a associação entre a personalidade e o bem-estar psicológico, verificando-se que um baixo neuroticismo aliado a uma alta extroversão, traduz a presença de níveis elevados de bem-estar psicológico. 0 bem-estar psicológico define-se ainda como um indicador de qualidade de vida, através da perceção positiva da autoestima, estabilidade emocional, profissional e familiar, traduzindo-se na satisfação do indivíduo com a sua vida no geral (Massé et.,1998). A autoestima implica a confiança, estima e amor que os indivíduos têm por si próprios; o equilíbrio prende-se com a presença de estabilidade em diversas esferas (pessoal, familiar e profissional) (Masselink, Van Roekel, \& Oldehinkel, 2017); o envolvimento social encontra-se associado às ambições, assim como à curiosidade em variadas atividades em geral, nomeadamente de lazer; a sociabilidade diz respeito ao relacionamento com a comunidade de forma empática; o controlo de si e dos acontecimentos associa-se à aptidão de encarar situações adversas de forma ajustada, por fim, a felicidade relaciona-se com sentimentos positivos em relação à vida em geral) (Gómez-Baya, et al. 2018; Massé et al,.1998). Os vínculos assumem um papel significativo no bem-estar psicológico (Collins \& Feeney, 2000), uma vez que o apoio conferido pelos pais conduz à satisfação global do indivíduo (Claes, 2004). Ainda o processo de separação-individuação, em que os pais são percecionados como base segura, possibilita aos jovens o desenvolvimento da sua autonomia (Fleming, 1996), em que a variabilidade desta se associa à satisfação global com a vida e o bem-estar psicológico (Sheldon, Ryan, Deci, \& Kasser, 2004).

A presente investigação tem como objetivo geral analisar de que forma os processos de separação individuação aos pais e a personalidade, predizem o bem-estar em jovens adultos. Pretende-se testar o efeito mediador da personalidade na associação separação-individuação aos pais e o bem-estar.

\section{MÉTODO}

\section{Participantes}

A amostra deste estudo é composta por 948 sujeitos (327 do sexo masculino - $34.5 \%$ e 621 do sexo feminino - 65.5\%), com idades compreendidas entre os 18 e os 30 anos de idade $(M=21.76, D P=3.31)$ e a frequentar o ensino superior na zona interior norte de Portugal. Dos 948 jovens adultos em estudo, no que diz respeito às relações, $493(52 \%)$ têm atualmente um relacionamento amoroso com uma duração média de 30,95 meses, correspondendo aproximadamente a 2 anos e 7 meses $(M=30.95, D P=33.30), 324$ (34.2\%) já tiveram uma relação amorosa, com uma duração média de 14.96 meses, correspondendo a aproximadamente 1 ano e 3 meses $)(M=14.96, D P=17.03), 129(13,6 \%)$ nunca tiveram algum tipo de relacionamento e 27 (2.8\%) mantêm um relacionamento amoroso, mas sem compromisso.

\section{Instrumentos}


Foi utilizado um Questionário de Dados Sociodemográficos, no sentido de obter informações pessoais acerca dos participantes e das figuras parentais.

Para avaliação da individuação aos pais utilizou-se o Individuation Test for Emerging Adults (ITEAKomidar, Zupančič, Sočan, \& Puklek Levpušček, 2013), adaptado por Correia \& Mota em 2016. 0 teste comporta 36 itens distribuídos em cinco dimensões avaliadas para o pai e mãe separadamente: suporte de si, ligação, intrusividade, autoconfiança e medo de desapontar os pais. Os itens apresentam-se numa escala de Likert através de uma gradação de 5 pontos (1-5) que varia entre "completamente falso" e "completamente verdadeiro". A análise da consistência interna do instrumento total para a mãe e para o pai, respetivamente, é de $.81 / .85$, e para as dimensões revelou valores de alfa de Cronbach: suporte de si= $.82 / .84 ;$ ligação $=.85 / .86 ;$ intrusividade $=.85 / .83$; autoconfiança $=.80 / .79 ;$ medo de desapontar os pais $=$ .77/.82. A análise fatorial confirmatória para a mãe e para o pai, respetivamente, apresenta índices de ajustamento com os seguintes valores: $\chi 2(53)=256.29 ; \chi 2(52)=314.82, p=.000 / .000, \mathrm{CFI}=.96 / .95$, $\mathrm{TLI}=.94 / .93, \mathrm{RMR}=.05 / .06, \mathrm{RMSEA}=.06 / .07$.

Para avaliação da personalidade utilizou-se o Inventário de personalidade dos Cinco Fatores (NEOFFI-20) (McCrae \& Costa, 1999), adaptado para a população portuguesa por Bertoquini \& Pais-Ribeiro em 2006. É constituído por 20 itens agrupados em cinco fatores: neuroticismo, abertura à experiência, amabilidade, conscienciosidade e extroversão. Os itens apresentam-se numa escala de Likert através de uma gradação de 5 pontos (1-5) que varia entre: "discordo fortemente" e "concordo fortemente". É pertinente salientar que alguns itens se encontram apresentados de forma invertida $(1,3,11,13,14,18$ e 19). Os itens 6 e 16 foram retirados do instrumento, uma vez que baixavam a sua consistência interna. 0 alfa de Cronbach para o instrumento na totalidade é de .51 e para cada uma das dimensões apresenta os seguintes valores: neuroticismo $=.75$, extroversão $=.66$, abertura à experiência $=.69$, amabilidade $=60$ e .75 para a conscienciosidade. A análise fatorial confirmatória do instrumento apresenta valores ajustados: $\chi 2(122)=3.588, \mathrm{p}=.000, \mathrm{CFI}=.917, \mathrm{TLI}=.896, \mathrm{RMR}=.0442, \mathrm{RMSEA}=.052$.

Para avaliação do bem-estar psicológico, utilizou-se a Escala de Medida de Manifestação de Bemestar psicológico (EMMBEP) (Massé, Poulin, Dassa, Lambert, Bélair, \& Battaglini,1998) adaptada para a população portuguesa por Monteiro, Tavares \& Pereira em 2006. Esta escala é composta por 25 itens agrupados em 6 fatores: auto- estima, equilíbrio, envolvimento social, sociabilidade, controlo de si e dos acontecimentos e felicidade. Os itens apresentam-se numa escala de likert através de uma gradação de cinco pontos (1-5) que varia entre: "nunca" e "quase sempre". A consistência interna para o instrumento na totalidade é de .93 e para as seis dimensões, apresenta os seguintes valores: felicidade $=.88$, controlo de si e dos acontecimentos $=.84$, sociabilidade $=.83$, envolvimento social=.69, autoestima=.84, e equilíbrio=.70. A análise fatorial confirmatória apresenta valores ajustados: $\chi 2(88)=430.82, p=.000, \mathrm{CFI}=.952, \mathrm{TLI}=.935$, $\mathrm{RMR}=.0405$, RMSEA $=.06$.

\section{Procedimentos}

Elaborou-se o protocolo, sendo submetido ao conselho de Ética da UTAD e aprovado com o n.ำ20/2016. Foram, à posteriori, solicitadas as autorizações às escolas da Universidade de Trás-os-Montes e Alto Douro para, assim, se iniciar a recolha dos dados. A recolha dos dados foi realizada na Universidade de Trás-osMontes e Alto Douro, bem como junto de jovens essencialmente da região norte do país. Realizou-se um consentimento informado onde foram abordados os objetivos gerais do estudo e todos os pontos referentes à participação voluntária e à confidencialidade da informação conferida.

\section{RESULTADOS}

Variância da separação-individuação aos pais, personalidade e bem-estar em função da idade, sexo e estado relacional dos jovens adultos

Foram realizadas análises de comparação de médias (Teste-t para amostras independentes) e análises de variância multivariada (MANOVA). Quanto à idade foram criados três grupos: grupo 1 (dos 18 aos 23 anos); grupo 2 (dos 24 aos 27 anos) e grupo 3 (dos 28 aos 30 anos), de forma a realizarem-se as análises diferenciais. Os resultados obtidos apontam a presença de diferenças estatisticamente significativas das figuras parentais face à idade, nomeadamente na dimensão autoconfiança, na mãe, $F(2,1882)=4.89, p=$ $.008, \mathrm{\eta}^{2}=.81$, destacando-se os jovens com idades compreendidas entre os 28 e os 30 anos $(M=3.60, D P=$ .74) comparativamente com os jovens com idades entre os 18 e os 23 anos $(M=3.37, D P=.77)$, com maiores níveis de autoconfiança, e no pai, $F(2,1882)=3.52, p=.030, \eta^{2}=.66$, apresentando os jovens com idades compreendidas entre os 28 e os 30 anos $(M=3.59, D P=.73)$ níveis mais elevados de autoconfiança em relação aos jovens com idades entre os 18 e os 23 anos $(M=3.39, D P=.77)$. Também na dimensão medo de desapontar, na mãe, $F(2,1882)=4.05, p=.018, \eta^{2}=.72$, evidenciando-se maiores níveis de medo de desapontar nos jovens com idades entre os 18 e 23 anos $(M=3.81, D P=.69)$ em relação aos jovens que 
compreendem idades entre os 24 e 27 anos $(M=3.64, D P=.67)$ e no pai, $F(2,1882)=6.78, p=.001, \eta^{2}=$ .92 , apresentando os jovens com idades entre os 18 e 23 anos $(M=3.67, D P=.81)$ níveis superiores de medo de desapontar quando comparados com os jovens que apresentam idades entre os 24 e 27 anos $(M$ $=3.44, D P=.74)$. A dimensão suporte de si, $F(2,1882)=4.15, \mathrm{p}=.016, \eta^{2}=.73$ apresenta diferenças significativas apenas na figura materna, sendo os jovens entre os 18 e 23 anos $(M=3.96, D P=.76)$ que apresentam maiores níveis de suporte de si quando comparados com os jovens que compreendem idades entre os 28 e 30 anos $(M=3.73, D P=.89)$. No que se refere à variável bem-estar psicológico, denotam-se diferenças estatisticamente significativas, especificamente na dimensão envolvimento social, $F(2,1880)=$ $6.93, p=.001, \eta^{2}=.93$, sendo que os jovens com idades entre os 24 e 27 anos $(M=3.90, D P=.68)$ apresentam maiores níveis de envolvimento social em relação aos jovens com idades entre os 18 e 23 anos $(M=3.70$, $D P=.72)$ e os jovens com idades entre os 28 e 30 anos $(M=3.89, D P=.67)$ evidenciam níveis maiores de envolvimento social comparados com os jovens que compreendem idades entre os 18 e 23 anos $(M=3.70$, $D P=.72$ ). No que diz respeito à variável personalidade, assinalaram-se diferenças estatisticamente significativas na dimensão neuroticismo, $F(2,1882)=3.48, p=.031, \eta^{2}=.65$, sendo que os jovens entre os 18 e 23 anos $(M=2.78, D P=.94)$ apresentam maiores níveis de neuroticismo quando comparados com os jovens com idades entre os 24 e 27 anos $(M=2.54, D P=.83)$. Também se verificam diferenças significativas na dimensão conscienciosidade, $F(2,1882)=12.82, p<.001, \eta^{2}=1$, sendo os jovens com idades entre os 28 e 30 anos $(M=4.17, D P=.51)$ que apresentam maiores níveis de conscienciosidade em relação aos jovens com idades compreendidas entre os 18 e os 23 anos $(M=3.90, D P=.56)$ e em relação também aos jovens com idades compreendidas entre os 24 e os 27 anos $(M=3.83, D P=.48)$ (Tabela 1$)$.

Tabela 1. Análise diferencial da separação-individuação aos pais, bem-estar e personalidade em relação à idade.

\begin{tabular}{|c|c|c|c|c|c|c|c|}
\hline & \multicolumn{2}{|c|}{ 1- dos 18 aos 23} & \multicolumn{2}{|c|}{ 2- dos 24 aos 27} & \multicolumn{2}{|c|}{ 3- dos 28 aos 30} & \multirow{2}{*}{$\begin{array}{c}\text { Direção } \\
\text { das } \\
\text { diferenças }\end{array}$} \\
\hline & $\mathbf{M} \pm \mathbf{D P}$ & IC $95 \%$ & $\mathbf{M} \pm \mathbf{D P}$ & IC $95 \%$ & $\mathbf{M} \pm \mathbf{D P}$ & IC $95 \%$ & \\
\hline \multicolumn{8}{|c|}{$\begin{array}{l}\text { Separação - individuação } \\
\text { Mãe }\end{array}$} \\
\hline Suporte de si & $3.96 \pm .76$ & {$[-.26, .10]$} & $3.88 \pm .76$ & {$[-.10, .26]$} & $3.73 \pm .89$ & {$[.03, .43]$} & $1>3$ \\
\hline Autoconfiança & $3.37 \pm .77$ & {$[-.07, .28]$} & $3.48 \pm .63$ & {$[-.28, .07]$} & $3.60 \pm .74$ & $\begin{array}{c}{[-.43,-} \\
.04]\end{array}$ & $1<3$ \\
\hline Medo de desapontar & $3.81 \pm .69$ & $\begin{array}{l}{[-.33,-} \\
.01]\end{array}$ & $3.64 \pm .67$ & {$[.01, .33]$} & $3.70 \pm .75$ & {$[-.07, .30]$} & $1>2$ \\
\hline \multicolumn{8}{|c|}{ Separação- individuação Pai } \\
\hline Autoconfiança & $3.39 \pm .77$ & {$[-.08, .27]$} & $3.49 \pm .64$ & {$[-.27, .08]$} & $3.59 \pm .73$ & {$[-.39, .00]$} & $1<3$ \\
\hline Medo de desapontar & $3.69 \pm .80$ & $\begin{array}{c}{[-.43,-} \\
.06]\end{array}$ & $3.44 \pm .74$ & {$[.06, .43]$} & $3.51 \pm .81$ & {$[-.03, .39]$} & $1>2$ \\
\hline \multicolumn{8}{|c|}{ Bem-estar psicológico } \\
\hline Envolvimento social & $3.70 \pm .72$ & {$[.04, .37]$} & $3.90 \pm .68$ & $\begin{array}{c}{[-.37,-} \\
.04]\end{array}$ & $3.89 \pm .67$ & $\begin{array}{c}{[-.38,-} \\
.01]\end{array}$ & $1<2 ; 1<3$ \\
\hline \multicolumn{8}{|l|}{ Personalidade } \\
\hline Conscienciosidade & $3.90 \pm .56$ & {$[-.19, .06]$} & $3.83 \pm .48$ & {$[-.06, .19]$} & $4.17 \pm .51$ & $\begin{array}{c}{[-.41,-} \\
.13]\end{array}$ & $1<3 ; 2<3$ \\
\hline Neuroticismo & $2.78 \pm .94$ & $\begin{array}{c}{[-.45,-} \\
.02]\end{array}$ & $2.54 \pm .83$ & {$[-.24, .25]$} & $2.77 \pm 1.05$ & {$[.02, .45]$} & $1>2$ \\
\hline
\end{tabular}

Relativamente à variável separação-individuação na mãe, os resultados obtidos apontam a presença de diferenças estatisticamente significativas relativamente ao sexo, nomeadamente nas dimensões: suporte de si $t(946)=-6.46, p=.000$, ligação $t(946)=-2.69, p=.007$, autoconfiança $t(775.55)=3.25, p$ $=.002$ e medo de desapontar $t(946)=-4.36, p<.001$, verificando-se que o sexo feminino evidencia maiores níveis de suporte de si $(M=4.04, D P=.75)$, ligação $(M=4.30, D P=.65)$ e medo de desapontar $(M=3.85, D P$ $=.67)$ em relação ao sexo masculino suporte de si $(M=3.71, D P=.77)$; ligação $(M=4.18, D P=.66)$; e medo de desapontar $(M=3.64, D P=.73)$. Por sua vez o sexo masculino apresenta maiores níveis de autoconfiança $(M=3.51, D P=.66)$ quando comparado com o sexo feminino $(M=3.36, D P=.79)$. Considerando a variável da separação-individuação no pai relativamente ao sexo, constatam-se diferenças significativas nas dimensões: intrusividade, $t(946)=2.16, p=.031$, autoconfiança, $t(756.31)=2.41, p=.016$, e medo de desapontar, $t(946)=-3.94, p<.001$, verificando-se que os rapazes apresentam maiores níveis de intrusividade $(M=2.55, D P=.81)$ e autoconfiança $(M=3.50, D P=.67)$ em relação às raparigas (intrusividade $(M=2.43, D P=.79)$; autoconfiança $(M=3.39, D P=.78)$ ). Por outro lado, as raparigas apresentam maiores níveis de medo de desapontar $(M=3.71, D P=.78)$ do que os rapazes $(M=3.49, D P=$ 
.83). Analisando as variáveis da personalidade face ao sexo, evidenciaram-se diferenças significativas nas dimensões neuroticismo, $t(946)=-4.53, p=.000$, extroversão $t(946)=3.31, p=.001$ e amabilidade, $t(946)$ $=-2.24, p=.025$, onde se constata que o sexo feminino apresenta maiores níveis de neuroticismo $(M=2.84$, $D P=.92)$ e amabilidade $(M=3.43, D P=.72)$ quando comparado com o sexo masculino (neuroticismo $(M=$ $2.56, D P=.95)$; amabilidade $(M=3.32, D P=.78)$ ). Por sua vez, analisando as médias, observa-se que o sexo masculino apresenta maiores níveis de extroversão $(M=3.76, D P=.65)$ do que o sexo feminino $(M=3.61$, $D P=.67)$. No que respeita às variáveis do bem-estar psicológico face ao sexo, denotam-se diferenças significativas nas dimensões autoestima, $t(946)=3.76, p<.001$, equilíbrio, $t(946)=-2.07, p=.039$, controlo de si e dos acontecimentos, $t(787.56)=4.55, p<.001$ e felicidade, $t(946)=3.96, p<.001$, evidenciando os rapazes maiores níveis de autoestima $(M=3.89, D P=.69)$, controlo de si e dos acontecimentos $(M=3.78$, $D P=.62)$ e felicidade $(M=3.84, D P=.67)$ quando comparados com as raparigas (níveis de autoestima $(M$ $=3.71, D P=.68)$, controlo de si e dos acontecimentos $(M=3.57, D P=.76)$ e felicidade $(M=3.65, D P=.72))$. Por outro lado, as raparigas apresentam maiores níveis de equilíbrio $(M=3.92, D P=.71)$ do que os rapazes $(M=3.82, D P=.75)$ (Tabela 2$)$.

Tabela 2. Análise diferencial da separação-individuação aos pais, bem-estar e personalidade em relação ao sexo.

\begin{tabular}{|c|c|c|c|c|c|}
\hline & \multicolumn{2}{|c|}{ 1-Masculino } & \multicolumn{2}{|c|}{ 2- Feminino } & \multirow{2}{*}{$\begin{array}{c}\text { Direção das } \\
\text { diferenças }\end{array}$} \\
\hline & $\mathbf{M} \pm \mathbf{D P}$ & IC 95\% & $\mathbf{M} \pm \mathbf{D P}$ & IC $95 \%$ & \\
\hline \multicolumn{6}{|c|}{$\begin{array}{l}\text { Separação - individuação } \\
\text { Mãe }\end{array}$} \\
\hline Suporte de si & $3.71 \pm .77$ & {$[-.436,-.233]$} & $4.04 \pm .75$ & {$[-.436,-.233]$} & $1<2$ \\
\hline Ligação & $4.18 \pm .66$ & {$[-.208, .033]$} & $4.30 \pm .65$ & {$[-.208, .033]$} & $1<2$ \\
\hline Autoconfiança & $3.51 \pm .66$ & {$[.062, .251]$} & $3.36 \pm 7.9$ & {$[.062, .251]$} & $1>2$ \\
\hline Medo de desapontar & $3.64 \pm .73$ & {$[-.299,-.113]$} & $3.85 \pm .67$ & {$[-.299,-.113]$} & $1<2$ \\
\hline \multicolumn{6}{|c|}{ Separação- individuação Pai } \\
\hline Intrusividade & $2.55 \pm .81$ & {$[.011, .224]$} & $2.43 \pm .79$ & {$[.011, .224]$} & $1>2$ \\
\hline Autoconfiança & $3.50 \pm .67$ & {$[.022, .212]$} & $3.39 \pm .78$ & {$[.022,-212]$} & $1>2$ \\
\hline Medo de desapontar & $3.49 \pm .83$ & {$[-.320,-.107]$} & $3.71 \pm .76$ & {$[-.320,-.107]$} & $1<2$ \\
\hline \multicolumn{6}{|l|}{ Bem-estar } \\
\hline Autoestima & $3.89 \pm .69$ & {$[.084, .268]$} & $3.71 \pm .68$ & {$[.084, .268]$} & $1>2$ \\
\hline Controlo de si & $3.78 \pm .62$ & {$[.118, .297]$} & $3.57 \pm .76$ & {$[.118, .297]$} & $1>2$ \\
\hline Felicidade & $3.84 \pm .67$ & {$[.096, .284]$} & $3.65 \pm .72$ & {$[.096, .284]$} & $1>2$ \\
\hline Equilíbrio & $3.82 \pm .75$ & {$[-.199,-.005]$} & $3.92 \pm .71$ & {$[-.199,-.005]$} & $1<2$ \\
\hline \multicolumn{6}{|l|}{ Personalidade } \\
\hline Neuroticismo & $2.56 \pm .95$ & {$[-.414,-.164]$} & $2.84 \pm .92$ & {$[-.414,-.164]$} & $1<2$ \\
\hline Amabilidade & $3.32 \pm .78$ & {$[-.214,-.014]$} & $3.43 \pm .72$ & {$[-.214,-.014]$} & $1<2$ \\
\hline Extroversão & $3.76 \pm .65$ & {$[.061, .238]$} & $3.61 \pm .67$ & {$[.061, .238]$} & $1>2$ \\
\hline
\end{tabular}

\section{Efeito da separação-individuação aos pais no bem-estar psicológico: papel mediador da} personalidade

Tendo em conta o objetivo de analisar o papel mediador, nomeadamente as caraterísticas da personalidade emocionalmente ajustada (extroversão, amabilidade, abertura à experiência e conscienciosidade) e o neuroticismo, foram tidas em consideração todas as relações possíveis entre as variáveis, nomeadamente as dimensões da separação-individuação na mãe e no pai e do bem-estar psicológico. Importa ressalvar que a dimensão abertura à experiência foi retirada deste procedimento estatístico, uma vez que na testagem inicial não apresenta qualquer predição significativa, tal como no modelo final com a introdução da variável mediadora.

Primeiramente, denotou-se um efeito inicial positivo da separação-individuação positiva na mãe no bem-estar psicológico $(\beta=.19)$. De igual modo, foi possível averiguar que a separação-individuação positiva na mãe prediz positivamente as caraterísticas ajustadas da personalidade $(\beta=.11)$. Posteriormente, verificou-se que a personalidade ajustada prediz de forma positiva o bem-estar $(\beta=.80)$ e, por sua vez, o 
neuroticismo prediz de forma negativa o bem-estar $(\beta=-.21)$. A intrusividade na figura materna não denotou resultados significativos na predição da personalidade e do bem-estar psicológico.

No modelo final, após introduzidas as variáveis mediadoras, observou-se que o efeito direto da separação-individuação positiva na mãe face ao bem-estar $(\beta=.19)$ perde a significância $(\beta=.00)$, observando-se uma mediação total positiva ( $\mathrm{SE}=.04, p=.200, \beta=.079$, IC 90\% [.02, .12]), onde a personalidade ajustada assume uma mediação total com efeito positivo na associação entre a separaçãoindividuação na mãe positiva e o bem-estar. 0 modelo apresenta os índices de ajustamento adequados $\chi^{2}$ $(100)=869.562, p<.001, \mathrm{CFI}=.84, \mathrm{SRMR}=.07, \mathrm{RMSEA}=.09$ (Figura 1$)$.

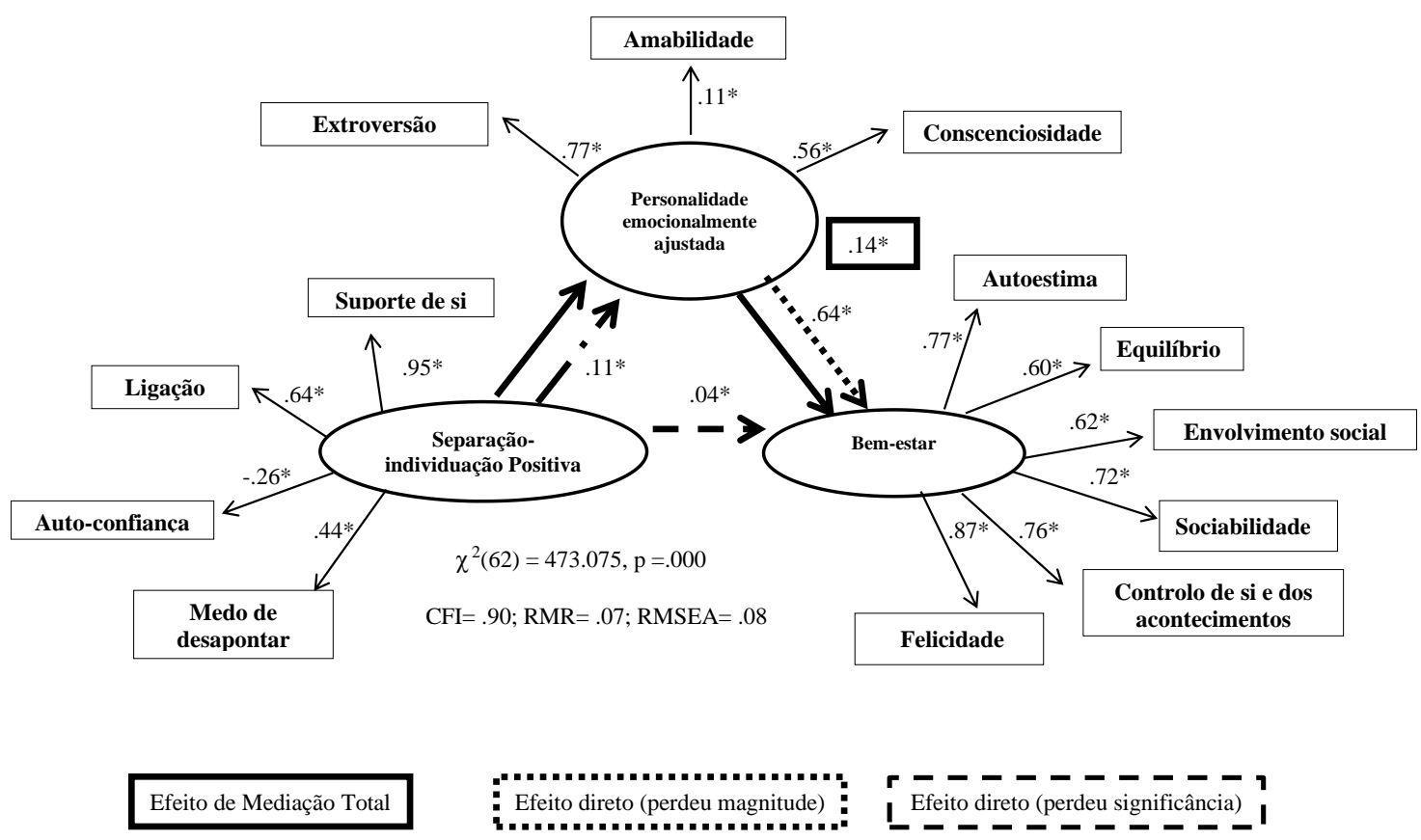

Figura 1. Papel mediador da personalidade emocionalmente ajustada na associação entre a separaçãoindividuação na mãe e o bem-estar

Relativamente à figura paterna, observou-se um efeito inicial negativo da separação-individuação no pai positiva face ao bem-estar psicológico $(\beta=-70)$. Foi passível ainda observar que a separaçãoindividuação positiva ao pai prediz positivamente as caraterísticas emocionalmente ajustadas da personalidade $(\beta=.16)$. Seguidamente, denotou-se que a personalidade ajustada prediz de forma positiva o bem-estar $(\beta=.80)$ e, por sua vez, o neuroticismo prediz de forma negativa o bem-estar $(\beta=-.21)$. A intrusividade na figura paterna não apresentou resultados significativos na predição da personalidade e do bem-estar psicológico.

No modelo final, após introduzidas as variáveis mediadoras, verificou-se que o efeito direto da separação-individuação positiva no bem-estar ( $\beta=-.70)$ mantém-se significativa, todavia perde magnitude $(\beta=.15)$, observando-se uma mediação parcial positiva (SE= .04, $p<.001, \beta=.190$, IC 90\% [.08, .22]). Neste sentido, a variável mediadora partilha o efeito da relação entre a variável dependente e independente, constatando-se que a personalidade ajustada explica parte do relacionamento entre as variáveis. Neste resultado está patente um efeito de supressão, uma vez que o efeito direto entre a variável independente e dependente apresentou sinais contrários (MacKinnon, Krull, \& Lockwood, 2000). É de referir que os índices de ajustamento se apresentaram adequados $(\chi 2(84)=739.334, \mathrm{p}=.000, \mathrm{CFI}=.87, \mathrm{SRMR}=.07, \mathrm{RMSEA}=$ .09 (Figura 2). 


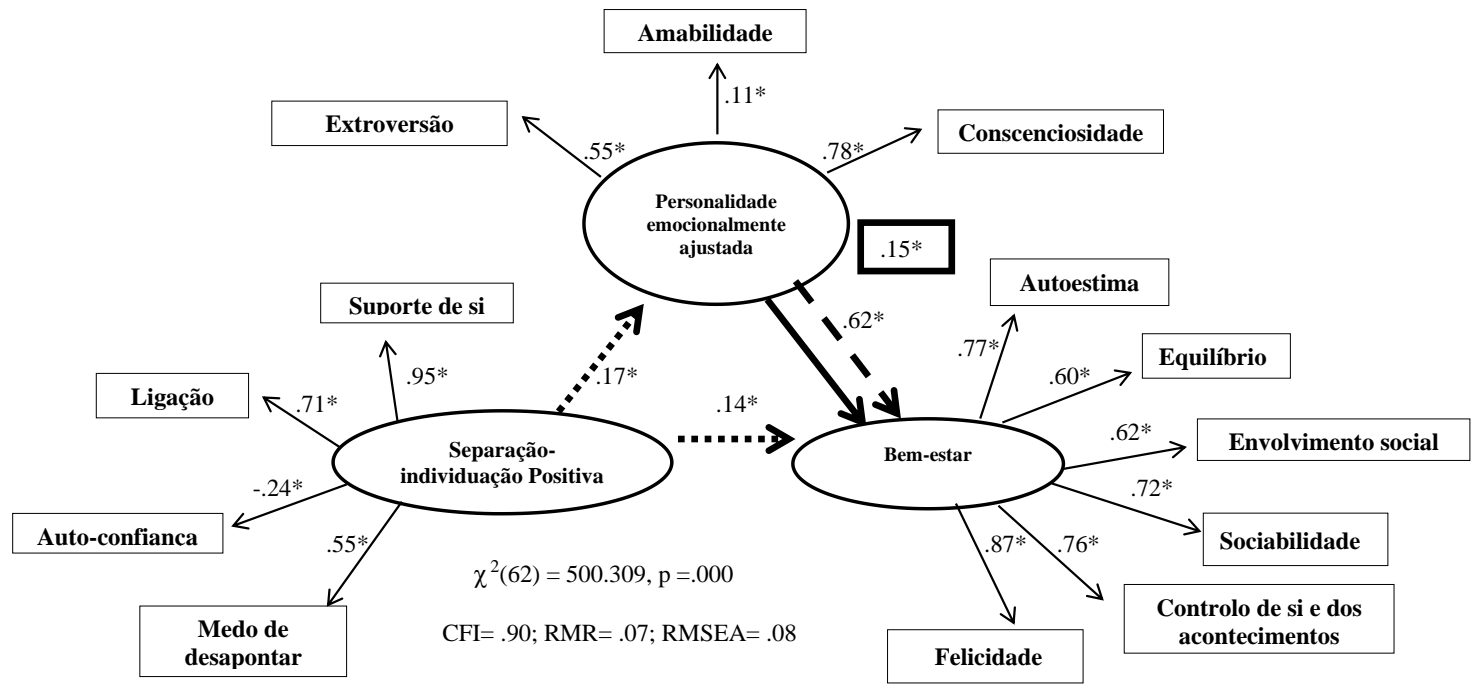

Efeito de Mediação Parcial

Figura 2. Papel mediador da personalidade emocionalmente ajustada na associação entre a separaçãoindividuação no pai e o bem-estar

\section{DISCUSSÃo}

O presente estudo teve como objetivo verificar o papel preditor da separação- individuação aos pais e da personalidade no bem-estar, bem como testar o papel mediador da personalidade na associação entre a separação-individuação aos pais e o bem-estar.

Os dados obtidos apontam diferenças significativas na separação-individuação face à idade, verificando-se em ambas as figuras parentais que os jovens com idades compreendidas entre os 28 e os 30 anos apresentam maiores níveis de autoconfiança em relação aos jovens com idades compreendidas entre os 18 e os 23 anos. 0 presente resultado é previsível, uma vez que à medida que aumenta a idade nos jovens, maior é a sua autoconfiança, caso desenvolvam o processo de individuação da melhor forma em relação às figuras parentais.

Koepke e Denissen (2012), Meeus et al., (2005) e Scabini (2000), defendem que o avançar da idade se assemelha a níveis maiores de autoconfiança, autonomia e individuação. Em ambas as figuras parentais observam-se diferenças na idade, uma vez que os jovens com idades compreendidas entre os 18 e os 23 anos apresentam maiores níveis de medo de desapontar em relação aos jovens que compreendem idades entre os 24 e os 27 anos, assim como na figura materna apresentam de igual modo maiores níveis de suporte de si. Mediante o resultado obtido, verifica-se que os jovens mais novos apontam uma maior apreensão em não responder às expetativas dos pais, assim como uma maior necessidade em apoiar-se na figura materna, podendo estes resultados serem justificados pelo facto de os jovens mais novos ainda se encontrarem dependentes a nível financeiro, assim como a nível emocional dos pais, daí resultar uma maior preocupação em desiludi-los. Sob a perspetiva de Zupančič e Kavčič (2014), o suporte nas figuras parentais fundamenta a preocupação sentida pelos jovens mais novos, uma vez que estes se apresentam menos maduros afetivamente e com menos aptidão para gerir as suas emoções. Em relação à variável bem-estar, verificam-se diferenças significativas face à idade, observando-se que os jovens com idades compreendidas entre os 24 e os 27 anos e entre os 28 e os 30 anos apresentam maiores níveis de envolvimento social em relação aos jovens com idades compreendidas entre os 18 e os 23 anos. A partir dos presentes dados, constata-se que os jovens adultos mais velhos apresentam um maior envolvimento em atividades novas, podendo ser justificado pelo facto de apresentarem maiores níveis de autonomia em relação aos pais. Schmitt, Long, McPhearson, O’Brien, Remmert, e Shah (2017) e Soto, John, Gosling e Potter (2011) comprovam que a participação em atividades e experiência novas, tende a crescer em paralelo com a idade. Em relação à variável personalidade, verificam-se diferenças significativas face à idade, observando-se que os jovens com idades compreendidas entre os 18 e os 23 anos apresentam maiores níveis de neuroticismo em relação aos jovens com idades compreendias entre os 24 e os 27 anos. A partir do resultado obtido, observa- se que os jovens mais novos apresentam mais caraterísticas neuróticas em relação aos jovens 
mais velhos, sendo justificado pelo facto de os jovens mais novos estarem a individualizar-se, sendo este um processo de inseguranças que inevitavelmente manifesta fragilidade emocional nos jovens. Alguns estudos comprovam que a extroversão está associada de forma negativa ao aumento da idade, uma vez que os sujeitos mais novos tendem a apresentar mais traços neuróticos (e.g. Lucas, \& Donnellan, 2009; Pusch, Mund, Hagemeyer, \& Finn, in press). Face à variância da conscienciosidade em relação à idade, os jovens com idades compreendidas entre os 28 e os 30 anos apresentam níveis maiores em relação aos jovens com idades compreendidas entre os 18 e os 23 anos e também em relação aos jovens com idades compreendidas entre os 24 e os 27 anos. Perante o seguinte resultado, em que os jovens mais velhos apresentam maiores níveis de consciência comparativamente com os mais novos, ele pode ser justificado pela maior maturidade emocional, daí apresentarem uma maior responsabilidade e ponderação (e.g. Allemand, Zimprich, \& Hendriks, 2008; Pusch, Mund, Hagemeyer, \& Finn, in press; Soto, et al., 2011). Um estudo de Anusic, Lucas e Donnellan (2012), com uma amostra de 7026 indivíduos, objetivou verificar as diferenças nas faixas etárias quanto à personalidade, chegando-se à conclusão de que a conscienciocidade se encontra correlacionada de forma positiva com a idade.

Os dados obtidos apontam ainda para diferenças significativas da separação- individuação na figura materna face ao sexo, em que o sexo feminino apresenta maiores níveis de suporte de si, ligação e medo de desapontar e o sexo masculino apresenta maiores níveis de autoconfiança. Já em relação à figura paterna, o sexo feminino apresenta maiores níveis de medo de desapontar e o sexo masculino maiores níveis de intrusividade e autoconfiança. A partir do presente resultado é percetível que as raparigas parecem apresentar uma maior necessidade de apoio e suporte em relação à figura materna, encontrando-se associada à preocupação em desiludir as figuras parentais. Segundo Gerlsma, Arrindell, Van der Veen e Emmerlkamp (1991), assumem que o suporte e proximidade emocional aos pais é percecionado de forma diferente por ambos os sexos, enfatizando que o sexo feminino tem uma perceção mais elevada e consistente do suporte das figuras parentais. Já os rapazes apresentam maiores níveis de autonomia em relação a ambas as figuras parentais, podendo ser fundamentado pelo facto de apresentarem menos dependência emocional em relação às figuras parentais, o que facilita a sua tomada de decisões de forma autónoma em relação às raparigas, uma vez que estas se encontram mais dependentes das figuras parentais. Os rapazes vão manifestando mais caraterísticas visíveis de autonomia comparativamente com as raparigas (Fleming, 1993). Importa ressaltar, ainda, que neste estudo os rapazes percecionam mais atitudes intrusivas por parte da figura paterna, comparativamente com as raparigas. Aqui seria expectável o contrário uma vez que os rapazes se mostram mais autónomos e independentes, sendo estas caraterísticas opostas às práticas intrusivas. Segundo Barber e Harmon (2002) a intrusividade e superproteção parental inibem e limitam de forma significativa autonomia e independência. Na personalidade observam-se diferenças no sexo, uma vez que as raparigas apresentam maiores níveis de neuroticismo e amabilidade, e os rapazes maiores níveis de extroversão. As raparigas demonstram-se mais neuróticas, tendendo a resultar desta vulnerabilidade a manifestação de emoções mais desajustadas como a ansiedade. Segundo alguns autores, as raparigas manifestam mais instabilidade emocional comparativamente com os rapazes (eg. Chapman, Duberstein, Sorensen, \& Lyness, 2007; Schmitt, et al. 2017; Schmitt, Realo, Voracek, \& Allik, 2008). Perante os dados obtidos, é possível ainda observar que as raparigas se mostram mais amáveis que os rapazes, podendo ser fundamentado pelo facto de as raparigas se apresentarem mais emotivas, mostrando com mais facilidade os seus sentimentos. Segundo alguns autores, as raparigas apresentam uma maior preponderância nos traços de amabilidade em comparação com os rapazes (Aluja, Garcia, Rossier, \& Garcia, 2005; Costa, Terracciano, \& McCrae, 2001). Em relação aos rapazes, estes revelam-se mais extrovertidos em relação às raparigas, podendo este resultado ser explicado pela maior sociabilidade com o meio à sua volta. Todavia, este resultado parece ser controverso uma vez que não é corroborado pela literatura. Segundo Weisberg, DeYoung e Hirsh (2011), em que examinaram num estudo com uma amostra de 2643 indivíduos com idades compreendidas entre os 17 e os 85 anos, tendo o intuito de examinar as diferenças entre o género e os traços de personalidade, observaram um maior peso da extroversão nas raparigas em comparação com os rapazes. E ainda o estudo de Vianello, Schnabel e Nosek (2013) com uma amostra de 14348 participantes com o intuito de avaliar as diferenças dos traços de personalidade no género, observou que as raparigas revelam maiores níveis de extroversão face aos rapazes. 0 bem-estar apresenta diferenças significativas em relação ao sexo, em que as raparigas apresentam maiores níveis de equilíbrio, e os rapazes maiores níveis de autoestima, controlo de si e dos acontecimentos e felicidade. Nos resultados obtidos, constata-se que as raparigas se mostram mais equilibradas em vários níveis, como profissional, pessoal, bem como familiar comparativamente com os rapazes, podendo ser explicado pelo facto de se caraterizarem como mais responsáveis e ponderadas. De acordo com a literatura, as raparigas manifestam valores mais elevados no que concerne à responsabilidade e sensatez, em comparação com os rapazes (Schmitt et al., 2008; Schmitt et al. 2017), sendo estas caraterísticas promotoras de um maior equilíbrio. Por outro lado, os rapazes mostram-se mais 
felizes e satisfeitos com a vida em geral, podendo o presente resultado ser aclarado pelo auto-conceito positivo que os rapazes manifestam em maior grau comparativamente com as raparigas. De acordo com Gómez-Baya, Lucia-Casademunt, \& Salinas-Pérez (2018), os rapazes manifestam mais sinais de felicidade e auto-aceitação, quando comparados com as raparigas, podendo ser justificado pelo facto de estes apresentarem mais estabilidade emocional e se mostrarem mais despreocupados com as diversas situações. Segundo vários autores, os rapazes revelam valores mais elevados e significativos na autoestima, quando comparados com as raparigas (e.g. Abella, 2003; Helwig, \&. Ruprecht, 2017; Masselink, Van Roekel, \& Oldehinkel 2017; Zimmerman, Copeland, Shope, \& Dielman, 1997). E, por fim, os rapazes na presente investigação revelam níveis superiores no controlo de situações de maior tensão, em relação às raparigas, parecendo assim relacionar-se com a perceção mais estável que estes têm acerca de si próprios, assim como com os níveis mais consistentes da satisfação com a vida. Segundo Masselink, et al. (2017), os indivíduos que manifestam mais autoestima, maior aceitação de si, apresentam-se mais seguros perante as dificuldades que surgem.

Finalmente, os resultados apontam para a personalidade emocionalmente ajustada, enquanto variável mediadora na associação entre a separação-individuação aos pais e o bem-estar psicológico, observando-se inicialmente que a separação-individuação na mãe positiva prediz positivamente o bemestar psicológico, por outro lado a separação-individuação no pai prediz negativamente o bem-estar psicológico. Este último resultado não era expectável, todavia na presente amostra poderá ser justificado pelo facto de os jovens adultos percecionarem a figura materna como uma figura que confere mais apoio e segurança, o que por sua vez facilita o processo de individuação que se reflete em níveis de bem-estar psicológico destes. Os jovens adultos que levam a cabo o processo de separação-individuação aos pais tendem a manifestar mais sinais de satisfação com a vida em geral. Pelo que, de acordo com a literatura, a satisfação global com a vida, o bem-estar psicológico, e a saúde mental, variam mediante o grau de autonomia (Gómez-Baya, et al., 2018; Sheldon et al., 2004). Também se constatou que a separaçãoindividuação nos pais positiva prediz as caraterísticas emocionalmente ajustadas da personalidade. Posto isto, foi possível constatar que a autonomia, independência e a individuação em relação aos pais contribui para a construção de uma personalidade saudável. Em consonância com a literatura, a estrutura de uma personalidade emocionalmente ajustada está associada às relações baseadas na prestação de apoio e cuidado no seio familiar (Bowlby, 1969) e, por sua vez, ao processo de separação-individuação (Kins, Soenens \& Beyers, 2011). Constatou-se ainda que a personalidade emocionalmente ajustada prediz positivamente as caraterísticas do bem-estar psicológico, ao passo que o neuroticismo prediz negativamente as dimensões do bem-estar psicológico. Perante o presente resultado, sugere-se que a manifestação de caraterísticas do bem-estar psicológico se deva sobretudo aos traços de personalidade emocionalmente ajustados. A personalidade encontra-se associada às caraterísticas do bem-estar psicológico (Ryff, 1989; Ryff \& Keyes, 1995; Novo, 2005), pelo que os traços emocionalmente ajustados da personalidade podem ser potenciadores de uma maior satisfação com a vida em geral que se assemelha ao bem-estar psicológico. Por outro lado, os traços emocionalmente disfuncionais acabam por inibir a manifestação de sinais de bem-estar psicológico (Gómez-Baya, et al. 2018). Em ambas as figuras parentais a separação-individuação positiva, assim como a intrusividade, não se mostraram preditoras do neuroticismo. Este resultado pode ser explicado pelo facto de as caraterísticas desajustadas da personalidade, como o caso do neuroticismo, poderem desenvolver-se independentemente da concretização do processo de separação-individuação aos pais, podem relacionar-se com fatores que têm mais peso como acontecimentos traumáticos que possam ter surgido ao longo da vida. E, ainda, na intrusividade não se verifica significância na sua predição face ao desenvolvimento da personalidade emocionalmente ajustada e o bem-estar psicológico. 0 presente achado pode ser justificado pelo facto de a intrusividade por parte dos pais não ter sido percecionada em grande escala pelos jovens da presente amostra, ao ponto de interferir negativamente no desenvolvimento da sua personalidade e por sua vez, do seu bem-estar psicológico.

No modelo final na figura materna observa-se que a personalidade emocionalmente ajustada assume um papel de mediação total na relação entre a separação-individuação na mãe positiva e o bem-estar psicológico. Já no modelo da figura paterna, observa-se uma mediação parcial, pelo que a relação entre a separação-individuação positiva no pai e o bem-estar é explicada parcialmente pela variável mediadora. Este resultado reflete a importância do ambiente familiar no desenvolvimento de determinados traços de personalidade que se traduzem em sinais de bem-estar psicológico. A personalidade emocionalmente ajustada parece clarificar o relacionamento da separação-individuação aos pais no desenvolvimento do bem-estar, assumindo um papel importante na associação entre a separação-individuação aos pais e o bemestar, explicando totalmente esta relação no caso da mãe e parcialmente no caso do pai. No caso do pai, com a introdução da variável mediadora, observou-se o efeito de supressão na relação da separaçãoindividuação positiva no pai e do bem-estar psicológico, uma vez que o sinal negativo da associação 
anterior passou a positivo, o que significa que a presença de caraterísticas emocionalmente ajustadas assume um papel positivo na associação mencionada, no sentido de o processo de separação- individuação bem conseguido, predizer positivamente as caraterísticas do bem-estar psicológico.

\section{Implicações práticas, limitações e pistas futuras}

A partir da investigação foi possível verificar que a qualidade dos vínculos com as figuras parentais e a separação-individuação aos pais levada a cabo com sucesso estão associadas ao desenvolvimento de traços de personalidade ajustados e aos sinais de bem-estar psicológico nos jovens adultos.

Ao longo do estudo observam-se algumas limitações referentes à presente investigação, primeiramente importa assinalar o facto de o presente estudo ser de caráter transversal, dificultando assim o estabelecimento de relações de causa e efeito entre as variáveis. A utilização de questionários de autorrelato, como método de recolha de dados é percecionada como outra limitação, uma vez que se verifica com mais facilidade a presença de desejabilidade social por partes dos jovens adultos. De realçar ainda, enquanto limitação, o desequilíbrio entre os participantes do sexo masculino e feminino, fruto da recolha realizada no contexto universitário. A presente investigação alerta para o papel relevante que as figuras parentais assumem no desenvolvimento saudável dos jovens adultos. É preponderante a concretização de futuros estudos longitudinais de modo a serem estudadas as relações de causalidade entre as variáveis em estudo. Seria também relevante em futuros estudos introduzir a variável estilos parentais e configuração familiar, com o objetivo de entender de que forma estes se associam ao desenvolvimento do processo de separação-individuação aos pais.

\section{REFERÊNCIAS}

Abella, M. (2003). Diferencias en autoestima en función del género. Análisis y Modificación de Conducta, 29(123), 51-78.

Ainsworth, M. D. S. (1967). Infancy in Uganda: Infant care and the growth of love. Johns Hopkins Press.

Ainsworth, M. S. (1989). Attachments beyond infancy. American Psychologist, 44(4), 709- 716.

Allemand, M., Zimprich, D., \& Hendriks, A. A. (2008). Age differences in five personality domains across the life span. Developmental Psychology, 44(3), 758- 770.

Aluja, A., Garcia, O., Rossier, J., \& Garcia, L. F. (2005). Comparasion of the NEO-FFI, the NEO-FFI-R and an alternative short version of the NEO-PI-R (NEO-60) in Swiss and Spanish samples. Personality and Individual Differences, 38(3), 591-604.

Anusic, I., Lucas, R. E., \& Donnellan, M. B. (2012). Cross-sectional age differences in personality: Evidence from nationally representative samples from Switzerland and the United States. Journal of Research in Personality, 46(1), 116-120. https://doi.org/10.1016/j.jrp.2011.11.002

Arnett, J. J. (2006). Emerging adulthood: Understanding the new way of coming of age. Emerging Adults in America: Coming of Age in the 21st century, 22, 3-19.

Arnett, J. J. (2007). Emerging adulthood: What is it, and what is it good for?. Child Development Perspectives, $1(2), 68-73$.

Barber, B. K. (2002). Intrusive parenting: How psychological control affects children and adolescents. American Psychological Association.

Barber, B. K., \& Harmon, E. L. (2002). Violating the self: Parental psychological control of children and adolescents. American Psychological Association.

Bertoquini, V., Ribeiro, J. (2006). Estudo de formas muito reduzidas do Modelo dos Cinco Factores da Personalidade. Psychologica, 43, 193-210.

Blos, P. (1996). O segundo processo de individuação. Artes Médicas.

Boles, S. A. (1999). A model of parental representations, second individuation, and psychological adjustment in late adolescence. Journal of Clinical Psychology, 55(4), 497-512.

Bowlby, J. (1969). Attachment and Loss: Vol.1. Attachment. Basic Books.

Bowlby, J. (1973). Attachment and Loss: Vol. 2. Separation: Anxiety and Anger. Basic Books.

Chapman, B. P., Duberstein, P. R., Sörensen, S., \& Lyness, J. M. (2007). Gender differences in Five Factor Model personality traits in an elderly cohort. Personality and Individual Differences, 43(6), 15941603. https://doi.org/10.1016/j.paid.2007.04.028

Claes, M. (2004). Les relations entre parents et adolescents: un bref bilan des travaux actuels. L'orientation Scolaire et Professionnelle, 33(2), 205-226.

Collins, N. L., \& Feeney, B. C. (2000). A safe haven: an attachment theory perspective on support seeking and caregiving in intimate relationships. Journal of Personality and Social Psychology, 78(6), 10531073. 
Correia, F., \& Mota, C. P. (2016). Ambiente familiar e qualidade da vinculação amorosa: Papel mediador da individuação em jovens adultos. Análise Psicológica, 34(1), 15-29. https://doi.org/10.14417/ap.1018

Costa Jr, P., Terracciano, A., \& McCrae, R. R. (2001). Gender differences in personality traits across cultures: robust and surprising findings. Journal of Personality and Social Psychology, 81(2), 322 - 331. https://doi.org/10.1037//0022-3514.81.2.322

Eisenberg, N., Fabes, R. A., Guthrie, I. K., \& Reiser, M. (2000). Dispositional emotionality and regulation: their role in predicting quality of social functioning. Journal of Personality and Social Psychology, 78(1), 136-157.

Fleming, M. (1993). Adolescência e autonomia: O desenvolvimento psicológico e a relação com os pais. Edições Afrontamento.

Fleming, M. (1996). Autonomia e adolescência. Edições Afrontamento.

Fleming, M. (2005). Entre o medo e o desejo de crescer: Psicologia da adolescência. Edições Afrontamento.

Gerlsma, C., Arrindell, W., Van der Veen, N., \& Emmerlkamp, P. (1991). A parental rearing style questionnaire for use with adolescentes: psychometric evaluation of the EMBU- A. Personality and Individual Diferences, 21, 1245-1252.

Gómez-Baya, D., Lucia-Casademunt, A. M., \& Salinas-Pérez, J. A. (2018). Gender differences in psychological well-being and health problems among European health professionals: Analysis of psychological basic needs and job satisfaction. International Journal of Environmental Research and Public Health, 15(7), 1474. https://doi.org/10.3390/ijerph15071474

Helwig, N. E., \&. Ruprecht, M. R. (2017). Age, gender, and self-esteem: A sociocultural look through a nonparametric lens. Archives of Scientific Psychology, 5, 19 -31. http://doi.org/10.1037/arc0000032

Kins, E., Soenens, B., \& Beyers, W. (2011). "Why do they have to grow up so fast?" Parental separation anxiety and emerging adults' pathology of separation- individuation. Journal of Clinical Psychology, 67(7), 647-664. https://doi.org/10.1002/j cl p.20786

Koepke, S., \& Denissen, J. J. (2012). Dynamics of identity development and separation- individuation in parent-child relationships during adolescence and emerging adulthood-A conceptual integration. Developmental Review, 32(1), 67-88. https://doi.org/10.1016/j.dr.2012.01.001

Kokko, K., Tolvanen, A., \& Pulkkinen, L. (2013). Associations between personality traits in psychological well-being across time in middle adulthood. Journal of Research in Personality, 47(6), 748-756.

Komidar, L., Zupančič, M., Sočan, G., \& Puklek Levpušček, M. (2013). Development and construct validation of the Individuation Test for Emerging Adults (IT-EA). Journal of Personality Assessment, 96(5), 1-12. https://doi.org/10.1080/00223891.2013.850703

Lucas, R. E., \& Donnellan, M. B. (2009). Age differences in personality: evidence from a nationally representative Australian sample. Developmental Psychology, 45(5), 1353-1363.

MacKinnon, D. P., Krull, J. L., \& Lockwood, C. M. (2000). Equivalence of the mediation, confounding and suppression effect. Prevention Science, 1(4), 1-13.

Mahler, M. S., Pine, F., \& Bergman, A. (1975). The Psychological Birth of the Human Infant. Symbiosis and Individuation. Basic Books.

Massé, R., Poulin, C., Dassa, C., Lambert, J., Bélair, S., \& Battaglini, A. (1998). Élaboration et validation d'un outil de mesure du bien-être psychologique: L'É.M.M.B.E.P. Revue Canadienne de Santé Publique, 89(5), 352-357.

Masselink, M., Van Roekel, E., \& Oldehinkel, A. J. (2017). Self-esteem in early adolescence as predictor of depressive symptoms in late adolescence and early adulthood: The mediating role of motivational and social factors. Journal of Youth and Adolescence, 47(5), 932-946. https://doi.org/10.1007/s10964-017-0727-z

McCrae, R. R., \& Costa Jr, P. T. (1999). The five-factor theory of personality. In L. Pervin, \& O. John (Eds.),

Handbook of Personality: Theory and Research.(p. 139-153). Guilford Press.

Meeus, W., Iedema, J., Maassen, G., \& Engels, R. (2005). Separation-individuation revisited: On the interplay of parent-adolescent relations, identity and emotional adjustment in adolescence. Journal of Adolescence, 28(1), 89- 106. https://doi.org/10.1016/j.adolescence. 2004.07.003

Monteiro, S., Tavares, J., \& Pereira, A. (2012). Adaptação portuguesa da escala de medida de manifestação de bem-estar psicológico com estudantes universitários - EMMBEP. Psicologia, Saúde \& Doenças, 13(1), 66-77.

Novo, R. (2005). Bem-estar e psicologia: conceitos e propostas de avaliação. Revista Iberoamericana de Diagnóstico y Evaluación-e Avaliação Psicológica, 2(20), 183- 203.

Nunes, C. H. S. S., \& Hutz, C. S. (2007). Construção e validação da escala fatorial de socialização no modelo dos cinco grandes fatores de personalidade. Psicologia:Reflexão e Crítica, 20(1), 20-25. 
Nunes, C. H. S. S., Hutz, C. S., \& Giacomoni, C. H. (2009). Associação entre bem-estar subjetivo e personalidade no modelo dos cinco grandes fatores. Avaliação Psicológica, 8(1), 99-108.

Pinto, M. C. (2009). Intimidade em adolescentes de diferentes grupos étnicos. Alto Comissariado para a Imigração e Diálogo Intercultural.

Pusch, S., Mund, M., Hagemeyer, B., \& Finn, C. (in press). Personality development in emerging and young adulthood: A study of age differences. European Journal of Personality. https://doi.org/10.1002/per.2181

Roberts, B. W. (2009). Back to the future: Personality and assessment and personality development. Journal of Research in Personality, 43(2), 137- 145. https://doi.org/10.1016/j.jrp.2008.12.015

Ryff, C. D. (1989). Hapiness is everything, or is it? Explorations on the meaning of psychological well-being. Journal of Personality and Social Psychology, 57 (6), 1069-1081.

Ryff, C. D., \& Keyes, C. L. M. (1995). The structure of psychological well-being revisited. Journal of Personality and Social Psychology, 69(4), 719-727.

Scabini, E. (2000). Parent-child relationships in Italian families: Connectedness and autonomy in the transition to adulthood. Psicologia: Teoria e Pesquisa, 16(1), 23-30.

Schmitt, D. P., Long, A. E., McPhearson, A., O’Brien, K., Remmert, B., \& Shah, S. H. (2017). Personality and gender differences in global perspective. International Journal of Psychology, 52, 45-56. https://doi.org/10.1002/ijop.12265

Schmitt, D. P., Realo, A., Voracek, M., \& Allik, J. (2008). Why can't a man be more like a woman? Sex differences in Big Five personality traits across 55 cultures. Journal of Personality and Social Psychology, 94(1), 168-182. https://doi.org/10.1037/0022-3514.94.1.168

Shanahan, M. J., Hill, P. L., Roberts, B. W., Eccles, J., \& Friedman, H. S. (2014). Conscientiousness, health, and aging: the life course of personality model. Developmental Psychology, 50(5), 1407-1425.

Sheldon, K. M., Ryan, R. M., Deci, E. L., \& Kasser, T. (2004). The independent effects of goal contents and motives on well-being: It's both what you pursue and why you pursue it. Personality and Social Psychology Bulletin, 30(4), 475-486. https://doi.org/10.1177/0146167203261883

Soto, C. J., John, O. P., Gosling, S. D., \& Potter, J. (2011). Age differences in personality traits from 10 to 65: Big Five domains and facets in a large cross-sectional sample. Journal of Personality and Social Psychology, 100(2), 330-348. https://doi.org/10.1037/a0021717

Vasconcellos, S. J. \& Hutz, C. S. (2008). Construção e validação de uma escala de abertura à experiência. Avaliação Psicológica, 7(2), 135-141.

Vianello, M., Schnabel, K., Sriram, N., \& Nosek, B. (2013). Gender differences in implicit and explicit personality traits. Personality and Individual Differences, 55(8), 994-999.

Weisberg, Y. J., DeYoung, C. G., \& Hirsh, J. B. (2011). Gender differences in personality across the ten aspects of the Big Five. Frontiers in Psychology, 2, 1-11. https://doi.org/10.3389/fpsyg.2011.00178

Wilkinson, R. B. (2004). The role of parental and peer attachment in the psychological health and selfesteem of adolescents. Journal of Youth and Adolescence, 33(6), 479-493. https://doi.org/00472891/04/1200-0479/0

Zimmerman, M. A., Copeland, L. A., Shope, J. T., \& Dielman, T. E. (1997). A longitudinal study of self-esteem: Implications for adolescent development. Journal of Youth and Adolescence, 26(2), 117-141.

Zupančič, M., \& Kavčič, T. (2014). Student personality traits predicting individuation in relation to mothers and fathers. Journal of Adolescence, 37, 715-726.

$\begin{array}{lr}\text { Historial do artigo } \\ \text { Recebido } & 11 / 2018 \\ \text { Aceite } & 12 / 2019 \\ \text { Publicado } & 08 / 2020\end{array}$

